\title{
Editorial
}

\section{Clinical significance of inflammatory markers of bacterial infection in critically ill patients with COVID-19 after treatment with anti-inflammatory and immunomodulatory drugs: a complex new scenario}

\author{
Daniele Roberto Giacobbe ${ }^{1,2, *}$, Lorenzo Ball ${ }^{3,4}$ Laura Magnasco $^{1}$, Chiara Dentone ${ }^{1}$, \\ Denise Battaglini ${ }^{3,5}$, Chiara Robba ${ }^{3,4}$, Paolo Pelosi ${ }^{3,4}$, Matteo Bassetti ${ }^{1,2}$ \\ ${ }^{1}$ Infectious Diseases Unit, San Martino Policlinico Hospital, IRCCS for Oncology and Neuroscience, 16132 Genoa, Italy, \\ ${ }^{2}$ Department of Health Sciences (DISSAL), University of Genoa, 16132 Genoa, Italy, ${ }^{3}$ Anesthesia and Intensive Care, San \\ Martino Policlinico Hospital, IRCCS for Oncology and Neuroscience, 16132 Genoa, Italy, ${ }^{4}$ Department of Surgical \\ Sciences and Integrated Diagnostics (DISC), University of Genoa, 16132 Genoa, Italy, ${ }^{5}$ Department of Medicine, \\ University of Barcelona, 08007 Barcelona, Spain
}

\section{TABLE OF CONTENTS}

\author{
1. Author contributions \\ 2. Ethics approval and consent to participate \\ 3. Acknowledgment \\ 4. Funding \\ 5. Conflict of interest \\ 6. References
}

Critically ill patients with severe hypoxemic respiratory failure due to coronavirus disease 2019 (COVID19) can develop severe secondary bacterial infections, that might contribute to unfavorably influencing their prognosis [1, 2]. A peculiar aspect of critically ill patients with COVID-19 is that almost all of them receive dexamethasone and some of them also receive interleukin-6 (IL-6) receptor antagonists (tocilizumab or sarilumab) or interleukin-1 receptor antagonists (anakinra). This practice is supported by the favorable results observed in randomized controlled trials (RCTs), in either the entire study population or specific subgroups, and is motivated by the contribution of an excessive host response to the organ damage in COVID-19 patients [3-8].

Besides their now established favorable effect on the course of the viral disease, the use of anti-inflammatory and immunomodulatory agents in critically ill patients with COVID-19 may nonetheless have some other relevant consequences: (i) an increased risk of bacterial infections [9]; (ii) a reduced ability of clinicians to promptly recognize these infections. While the first point is inherently acceptable in balance with the favorable effect on the viral disease if pragmatically considering the overall favorable results observed in RCTs [3-5], the second, i.e., reduced ability of clinicians to promptly recognize such infections, deserves a more in-depth discussion. For example, while it has been long known among immunologists and rheumatologists that patients treated with IL-6 receptor antagonists could develop bacterial infections without or with only mild increases in classical inflammatory markers [10], the use of such agents in critically ill patients was extremely rare before the COVID-19 era. Thus, an excess in the confidence on the reliability of inflammatory markers may slow down the recognition of possible secondary bacterial infections, with perilous delays in diagnosis and treatment.

Against this background, some published experiences have already highlighted the reduced value of serum C-reactive protein (CRP) and procalcitonin (PCT) for suggesting the presence of bacterial infections in critically ill patients with COVID-19. In a single center study in 2 intensive care units we observed that among 78 critically ill patients with COVID-19 who developed bloodstream infection, the median serum CRP values on the day the first positive blood culture was drawn was 169.0 $\mathrm{mg} / \mathrm{L}$ (interquartile range [IQR] 70.4-194.0), $105.2 \mathrm{mg} / \mathrm{L}$ (IQR 54.0-164.0), $20.6 \mathrm{mg} / \mathrm{L}$ (IQR 8.4-33.6), and 44.6 $\mathrm{mg} / \mathrm{L}$ (IQR 11.3-137.0) in patients who did not receive neither steroids nor tocilizumab, patients treated with steroids and not tocilizumab, patients treated with tocilizumab and not steroids, and patients treated with both steroids and tocilizumab, respectively [11]. The same was observed for PCT, with median values $>1 \mathrm{ng} / \mathrm{mL}$ being observed only in 


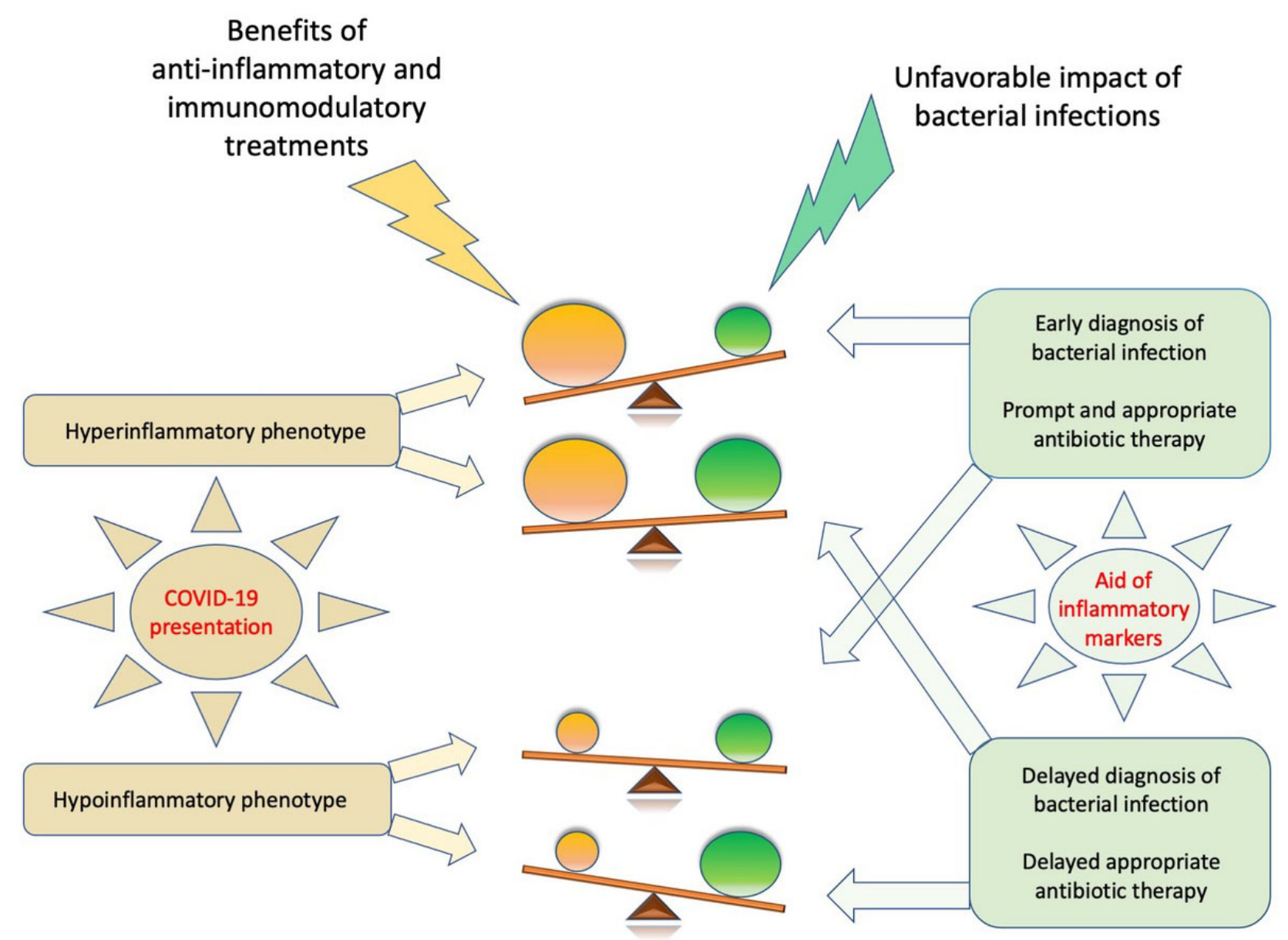

Fig. 1. Complex balance between the effects of anti-inflammatory/immunomodulatory agents for the treatment of COVID-19 and the possible unfavorable impact of bacterial infections.

patients who did not receive steroids nor tocilizumab [11]. These results are in line both with subsequently updated data from our center and with a large multicenter study conducted by Kooistra and colleagues in a cohort of 123 critically ill patients with COVID-19, of whom 71 developed bacterial infection (pulmonary infection or bloodstream infection) $[12,13]$. In patients treated with dexamethasone and tocilizumab in the study by Kooistra and colleagues, CRP and PCT values were similar between those who developed bacterial infection and those who did not, after adjustment for intensive care unit exposure up to development of infection. For PCT (but not for CRP) a statistically significant increase was observed by day 2 of infection in patients treated with dexamethasone and tocilizumab who developed bacterial infection, that, although remaining useful for identifying a previously undetected infection, may still imply perilous delays in diagnosis and treatment [12]. In this context, it is of note that serial PCT measurements have been proposed by some authors, although still without solid supporting evidence and deserving further investigation especially in patients treated with anti-inflammatory and/or immunomodulatory agents [14-16].
The interpretation of these results in the context of clinical practice is not easy. Indeed, on the surface, there could be the temptation to disregard the results of CRP and PCT assays, deeming them as useless for identifying bacterial infections in critically ill patients with COVID-19 (owing to the nonnegligible risk of false negative results). On the other hand, this would likely lead to a widespread use of empirical antibacterials guided only by clinical or radiological signs of infection, that are shared with the baseline viral disease. In turn, this could unacceptably increase both the rate of misdiagnosis and the selective pressure for antibacterial resistance. For this reason, we feel an alternative view of the results of the studies discussed above may be more appropriate from a clinical standpoint. First, the fact that CRP and PCT were low or negative at the onset of bacterial infection in many patients treated with steroids/tocilizumab does not mean that they were negative in all patients, as appreciable by carefully analyzing the results of the studies discussed above. We think this could be sufficient to continue measuring CRP and PCT in critically ill patients with COVID-19 and a clinical suspicion of bacterial infection, since in some patients their in- 
creased values may still be very useful for increasing the post-test probability, prompting a reasonable early use of empirical antibacterials while waiting for microbiological results. Of course, the core problem lies in the fact that many other critically ill COVID-19 patients with bacterial infection will have low/negative CRP and PCT values, at least at the onset of infection. In such cases, given the similarity of clinical and radiological signs of infection between worsening of COVID-19 and bacterial infections, we strongly believe the most appropriate solution would be that of potentiating our ability to rapidly obtain an etiological diagnosis. The proper use of rapid molecular or phenotypical tests for identifying bacteria (and some resistance determinants) in the blood and respiratory tract has the potential to provide valuable insights for distinguishing the worsening of the viral disease from a bacterial infection in critically ill patients with COVID-19 treated with antiinflammatory and immunomodulatory agents $[17,18]$. In turn, this would allow either a more targeted antibacterial treatment or the early discontinuation of broad-spectrum empirical antibiotics when a secondary infection is rapidly excluded. Furthermore, the importance of obtaining lower respiratory tract specimens for the microbiological diagnosis of ventilator-associated bacterial pneumonia should be stressed [19]. A putative role for biomarkers of bacterial infections in the complex context influencing the overall outcome of critically ill patients with COVID-19 is displayed in Fig. 1 [20-23]. Finally, although they were not the topic of the present editorial (focused on the role of biomarkers in bacterial infections), it should be reminded that critically ill patients with COVID-19 may also be at increased risk of fungal infections, and the use of tocilizumab has been recently recognized as an independent risk factor for COVID19-associated pulmonary aspergillosis (CAPA) [24].

In summary, during these almost two years of COVID-19 pandemic we have been forced on several occasions to rely only on very aspecific clinical and radiological signs for the diagnosis of bacterial infections in critically ill patients with COVID-19 treated with anti-inflammatory and immunosuppressive agents, due to the reduced value of CRP and PCT in this peculiar context. We firmly believe their use should be coupled with improved etiological diagnosis of bacterial infection if we were to follow the core principles of antimicrobial stewardship also in this complex new scenario.

\section{Author contributions}

DRG, LB, CD, LM, CR, DB, PP and MB conceived the project; $\mathrm{MB}, \mathrm{PP}$ and $\mathrm{DRG}$ supervised the project; DRG and LB drafted the manuscript; DRG, LB, CD, LM, $\mathrm{CR}, \mathrm{DB}, \mathrm{PP}$ and $\mathrm{MB}$ critically reviewed the manuscript for important intellectual content.

\section{Ethics approval and consent to participate}

Not applicable.

\section{Acknowledgment}

Thanks to all the peer reviewers for their opinions and suggestions.

\section{Funding}

This research received no external funding.

\section{Conflict of interest}

Outside the submitted work, DRG reports honoraria from Stepstone Pharma GmbH and unconditional grants from MSD Italia, Correvio Italia, and Pfizer Inc. Outside the submitted work, MB has received funding for scientific advisory boards, travel, and speaker honoraria from Angelini, Astellas, AstraZeneca, Basilea, Bayer, BioMeŕieux, Cidara, Correvio, Cubist, Menarini, Molteni, MSD, Nabriva, Paratek, Pfizer, Roche, Shionogi, Tetraphase, Thermo Fisher, and The Medicine Company. All the other authors have no conflicts of interest to disclose.

\section{References}

[1] Giacobbe DR, Battaglini D, Enrile EM, Dentone C, Vena A, Robba C, et al. Incidence and Prognosis of Ventilator-Associated Pneumonia in Critically Ill Patients with COVID-19: A Multicenter Study. Journal of Clinical Medicine. 2021; 10: 555.

[2] d'Humieres C, Patrier J, Lortat-Jacob B, Tran-Dinh A, Chemali L, Maataoui N, et al. Two original observations concerning bacterial infections in COVID-19 patients hospitalized in intensive care units during the first wave of the epidemic in France. PLoS ONE. 2021; 16: e0250728.

[3] RECOVERY Collaborative Group. Tocilizumab in patients admitted to hospital with COVID-19 (RECOVERY): a randomised, controlled, open-label, platform trial. Lancet. 2021; 397: 1637-1645.

[4] RECOVERY Collaborative Group, Horby P, Lim WS, Emberson JR, Mafham M, Bell JL, et al. Dexamethasone in Hospitalized Patients with Covid-19. New England Journal of Medicine. 2021; 384: 693-704.

[5] REMAP-CAP Investigators, Gordon AC, Mouncey PR, AlBeidh F, Rowan KM, Nichol AD, et al. Interleukin-6 Receptor Antagonists in Critically Ill Patients with Covid-19. New England Journal of Medicine. 2021; 384: 1491-1502.

[6] Bassetti M, Ansaldi F, Icardi G, Pelosi P, Robba C, Taramasso L, et al. COVID-19: Some clinical questions after the first 4 months. European Journal of Clinical Investigation. 2020; 50: e13326.

[7] Bassetti M, Giacobbe DR, Bruzzi P, Barisione E, Centanni S, Castaldo N, et al. Clinical Management of Adult Patients with COVID-19 Outside Intensive Care Units: Guidelines from the Italian Society of Anti-Infective Therapy (SITA) and the Italian Society of Pulmonology (SIP). Infectious Diseases and Therapy. 2021. 
[8] Kyriazopoulou E, Poulakou G, Milionis H, Metallidis S, Adamis G, Tsiakos K, et al. Early treatment of COVID-19 with anakinra guided by soluble urokinase plasminogen receptor plasma levels: a double-blind, randomized controlled phase 3 trial. Nature Medicine. 2021.

[9] Buetti N, Ruckly S, de Montmollin E, Reignier J, Terzi N, Cohen $\mathrm{Y}$, et al. COVID-19 increased the risk of ICU-acquired bloodstream infections: a case-cohort study from the multicentric OUTCOMEREA network. Intensive Care Medicine. 2021; 47: 180-187.

[10] Bari SF, Khan A, Lawson T. C reactive protein may not be reliable as a marker of severe bacterial infection in patients receiving tocilizumab. BMJ Case Reports. 2013; 2013: bcr2013010423.

[11] Giacobbe DR, Battaglini D, Ball L, Brunetti I, Bruzzone B, Codda G, et al. Bloodstream infections in critically ill patients with COVID-19. European Journal of Clinical Investigation. 2020; 50: e13319.

[12] Kooistra EJ, van Berkel M, van Kempen NF, van Latum CRM, Bruse N, Frenzel T, et al. Dexamethasone and tocilizumab treatment considerably reduces the value of C-reactive protein and procalcitonin to detect secondary bacterial infections in COVID19 patients. Critical Care. 2021; 25: 281.

[13] Taramasso L, Magnasco L, Portunato F, Briano F, Vena A, Giacobbe DR, et al. Clinical presentation of secondary infectious complications in COVID-19 patients in intensive care unit treated with tocilizumab or standard of care. European Journal of Internal Medicine. 2021.

[14] De Waele JJ, Derde L, Bassetti M. Antimicrobial stewardship in ICUs during the COVID-19 pandemic: back to the 90s? Intensive Care Medicine. 2021; 47: 104-106.

[15] Powell N, Howard P, Llewelyn MJ, Szakmany T, Albur M, Bond SE, et al. Use of Procalcitonin during the First Wave of COVID19 in the Acute NHS Hospitals: A Retrospective Observational Study. Antibiotics. 2021; 10: 516.

[16] Schouten J, De Waele J, Lanckohr C, Koulenti D, Haddad N, Rizk N, et al. Antimicrobial stewardship in the ICU in COVID19 times: the known unknowns. International Journal of Antimicrobial Agents. 2021; 58: 106409.

[17] Dubourg G, Lamy B, Ruimy R. Rapid phenotypic methods to improve the diagnosis of bacterial bloodstream infections: meeting the challenge to reduce the time to result. Clinical Microbiology and Infection. 2018; 24: 935-943.
[18] Fontana C, Favaro M, Minelli S, Bossa MC, Altieri A. Coinfections observed in SARS-CoV-2 positive patients using a rapid diagnostic test. Scientific Reports. 2021; 11: 16355.

[19] Pickens CO, Gao CA, Cuttica MJ, Smith SB, Pesce LL, Grant RA, et al. Bacterial Superinfection Pneumonia in Patients Mechanically Ventilated for COVID-19 Pneumonia. American Journal of Respiratory and Critical Care Medicine. 2021.

[20] Brodin P. Immune determinants of COVID-19 disease presentation and severity. Nature Medicine. 2021; 27: 28-33.

[21] Calabrese EJ, Kozumbo WJ, Kapoor R, Dhawan G, Lara PC Giordano J. Nrf2 activation putatively mediates clinical benefits of low-dose radiotherapy in COVID-19 pneumonia and acute respiratory distress syndrome (ARDS): Novel mechanistic considerations. Radiotherapy and Oncology. 2021; 160: 125-131.

[22] Schultze JL, Aschenbrenner AC. COVID-19 and the human innate immune system. Cell. 2021; 184: 1671-1692.

[23] Shi Y, Wang Y, Shao C, Huang J, Gan J, Huang X, et al. COVID19 infection: the perspectives on immune responses. Cell Death and Differentiation. 2020; 27: 1451-1454.

[24] Prattes J, Wauters J, Giacobbe DR, Salmanton-Garcia J, Maertens J, Bourgeois M, et al. Risk factors and outcome of pulmonary aspergillosis in critically ill coronavirus disease 2019 patients- a multinational observational study by the European Confederation of Medical Mycology. Clinical Microbiology and Infection. 2021.

Abbreviations: COVID-19, coronavirus disease 2019; CRP, C-reactive protein; IQR, interquartile range; PCT, procalcitonin; RCTs, randomized controlled trials.

Send correspondence to: Daniele Roberto Giacobbe, Infectious Diseases Unit, San Martino Policlinico Hospital, IRCCS for Oncology and Neuroscience, 16132 Genoa, Italy, Department of Health Sciences (DISSAL), University of Genoa, 16132 Genoa, Italy, E-mail: danieleroberto.giacobbe@unige.it 